\title{
Low Risk of Serological Cross-Reactivity between the Dengue Virus and SARS-CoV-2-IgG Antibodies Using Advanced Detection Assays
}

\author{
Farah M. Shurrab ${ }^{a}$ Duaa W. Al-Sadeq ${ }^{a, b}$ Fatima Humaira Amanullah ${ }^{a}$ \\ Enas S. Al-Absi ${ }^{a}$ Hamda Qotbac Hadi M. Yassine ${ }^{a, d}$ Laith J. Abu-Raddad ${ }^{\text {e, }}$, g \\ Gheyath K. Nasrallah ${ }^{a, d}$

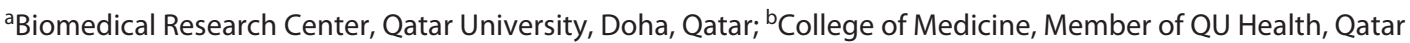 \\ University, Doha, Qatar; 'Department of Clinical Research, Primary Health Care Centers, Doha, Qatar; ${ }^{\mathrm{d} D e p a r t m e n t}$ \\ of Biomedical Science, College of Health Sciences, Member of QU Health, Qatar University, Doha, Qatar; ${ }^{\text {Infectious }}$ \\ Disease Epidemiology Group, Weill Cornell Medicine-Qatar, Cornell University, Qatar Foundation - Education \\ City, Doha, Qatar; ${ }^{\text {} W o r l d ~ H e a l t h ~ O r g a n i z a t i o n ~ C o l l a b o r a t i n g ~ C e n t r e ~ f o r ~ D i s e a s e ~ E p i d e m i o l o g y ~ A n a l y t i c s ~ o n ~ H I V / ~}$ \\ AIDS, Sexually Transmitted Infections, and Viral Hepatitis, Weill Cornell Medicine - Qatar, Cornell University, Qatar \\ Foundation - Education City, Doha, Qatar; ${ }^{9}$ Department of Healthcare Policy and Research, Weill Cornell Medicine, \\ Cornell University, New York, NY, USA
}

\section{Keywords}

Cross-reaction · Dengue $\cdot$ Severe acute respiratory syndrome coronavirus 2 . Chemiluminescence

immunoassay · Enzyme-linked immunosorbent assay

\begin{abstract}
Several studies have reported serological cross-reactivity of the immune responses between SARS-CoV-2 and DENV. Most of the available studies are based on the point-of-care rapid testing kits. However, some rapid test kits have low specificity and can generate false positives. Hence, we aimed to investigate the potential serological cross-reactivity between SARS-CoV-2 and DENV-IgG antibodies using advanced assays including chemiluminescence immunoassay (CLIA) and enzyme-linked immunosorbent assay (ELISA) test. A total of 90 DENV-lgG-ELISA-positive and 90 DENV-IgGELISA-negative prepandemic sera were tested for anti-SARSCoV-2-IgG using the automated CL-900i CLIA assay. Further-
\end{abstract}

Karger@karger.com www.karger.com/int

Karger $\stackrel{\text { ' }}{5}$

GOPEN ACCESS
(C) 2022 The Author(s)

Published by S. Karger AG, Basel

This is an Open Access article licensed under the Creative Commons Attribution-NonCommercial-4.0 International License (CC BY-NC) (http://www.karger.com/Services/OpenAccessLicense), applicable to the online version of the article only. Usage and distribution for commercial purposes requires written permission. more, a total of 91 SARS-CoV-2-IgG-CLIA-positive and 91 SARS-CoV-2-lgG-CLIA-negative postpandemic sera were tested for anti-DENV-IgG using the NovaLisa ELISA kit. The DENV-IgG-positive sera resulted in five positives and 85 negatives for SARS-CoV-2-IgG. Similarly, the DENV-IgG-negative sera also resulted in 5 positives and 85 negatives for SARSCoV-2-lgG. No statistically significant difference in specificity between the DENV-IgG-positive and DENV-IgG-negative sera was found ( $p$ value $=1.00$ ). The SARS-CoV-2-lgG-positive sera displayed 43 positives, 47 negatives, and 1 equivocal for DENV-IgG, whereas the SARS-CoV-2-IgG-negative sera resulted in 50 positives, 40 negatives, and 1 equivocal for DENV-IgG. No statistically significant difference in the proportion that is DENV-IgG positive between the SARS-CoV2-lgG-positive and SARS-CoV-2-lgG-negative sera ( $p$ value $=$ 0.58 ). In conclusion, there is a low risk of serological crossreactivity between the DENV and SARS-CoV-2-IgG antibodies when using advanced detection assays.

(c) 2022 The Author(s).

Published by S. Karger AG, Basel
Correspondence to:

Laith J. Abu-Raddad, lja2002@ qatar-med.cornell.edu Gheyath K. Nasrallah, gheyath.nasrallah@qu.edu.qa 


\section{Introduction}

The coronavirus disease 19 (COVID 19) is a respiratory disease caused by the severe acute respiratory syndrome coronavirus 2 (SARS-CoV-2), a highly infectious virus that originated from Wuhan, China, in late 2019 and has spread worldwide, resulting in a global pandemic. The impact of the COVID-19 pandemic was unprecedented on healthcare systems, the global economy, and resources [1]. Several reports demonstrated that crossreactivity occurs between anti-SARS-CoV-2 antibodies and human tissues [2, 3]. For instance, Vojdani et al. [2] showed that SARS-CoV-2 antibodies had reactions with 28 out of 55 tissue antigens, representing a diversity of tissue groups that included barrier proteins; gastrointestinal, thyroid, and neural tissues; and more. Others have also reported cross-reactivity of anti-SARS-CoV-2 antibodies and other viruses such as anti-dengue and influenza virus antibodies $[4,5]$.

Some countries faced challenges in the co-occurrence of SARS-CoV-2 and other endemic viruses like dengue virus (DENV), which increased the problem. DENV is an RNA virus from the Flaviviridae family; it has four genetically and antigenically serotypes (DENV-1, DENV-2, DENV-3, and DENV-4). The virus is transmitted to humans via a bite of an infected mosquito, usually from $A e-$ des aegypti and Aedes albopictus species, causing dengue disease [6]. According to the World Health Organization (WHO), dengue disease is a major health concern in many regions, including Africa, the Americas, the Eastern Mediterranean, South-East Asia, and the Western Pacific [7]. The simultaneous occurrence of COVID-19 pandemic and dengue disease in DENV endemic countries has doubled the burden on the healthcare system in these regions, having to face two complex diseases with shared clinical and diagnostic features [8].

The clinical symptoms of dengue and COVID-19 are similar, including cough, fever, skin rash, and fatigue, which makes it challenging to differentiate between the two viral infections and might lead to misdiagnosis [9]. These observations raised a lot of speculations regarding the possibility that preexposure to DENV might provide cross-protection immunity to SARS-CoV-2 infection [8]. That is, SARS-CoV-2 has antigenic similarity to DENV and elicits antibodies that are detected by DENV serological tests. The first reported cases of serological crossreactivity between COVID-19 and DENV were from Singapore. These cases were DENV-IgM and IgG false positive by rapid lateral flow assay (LFA). However, further testing of the original samples showed that the patients were negative for DENV by RT-PCR, and a repeat DENV rapid test was also negative, but RT-PCR positive for SARS-COV-2. Thus, the initial DENV seroconversion results were considered false positive [10].

Several other studies have reported serological crossreactivity of the immune responses between SARS-CoV-2 and DENV $[2,9]$. Most of the available studies are based on point-of-care rapid testing kits [11-14]. However, some rapid test kits have low specificity and can generate false positives [15]. Therefore, we aimed to investigate potential serological cross-reactivity between SARSCoV-2 and DENV-IgG antibodies using advanced assays including chemiluminescence immunoassay and enzyme-linked immunosorbent assay (ELISA) test.

\section{Methods}

\section{Study Design}

A total of 91 SARS-CoV-2-IgG-positive and 91 IgG-negative postpandemic sera confirmed by RT-PCR (total $=182$ ) were available from a recent study [16]. In addition, 90 DENV-IgG sero confirmed positive and $90 \mathrm{DENV}$-IgG sero confirmed negative (total $=180$ ) were available from a study conducted before the SARSCoV-2 pandemic [17]. Therefore, the 91 SARS-CoV-2-negative sera and the 90 DENV-negative sera were selected as the control group for the ELISA and the automated analyzer assay testing. Specimens were collected from males, 24-69 years of age, and nationalities of African, Asian, and Middle Eastern origins, all of them residing currently in Qatar.

\section{Study Participants}

Complete descriptions of the origin of the samples can be found in these articles [18]. These were an opportunistic crosssectional study on blood donor volunteers from different nationalities attending the Blood Donation Center at Hamad Medical Corporation, the main healthcare provider in Qatar, between June 2013 and June 2017. Blood donation in Qatar is a common practice, and individuals from diverse socioeconomic strata participate in blood donation campaigns. Round 6,000 blood donors consented to provide blood specimens and basic demographic information, including sex, age, and nationality. No identifiable information was collected. The research work was approved by the Ethics Boards and Research Committees at Hamad Medical Corporation and Qatar University. The samples contain Qataris and expatriates (MENA and non-MENA nationals) residing in Qatar, adults $\geq 18$ years of age. The number of female participants was small, and consequently, women were excluded. Positive and negative antidengue samples used in this study were randomly selected based on the results of our previous publication, where we used the above male samples for studying dengue and chikungunya seroprevalence among male Qatari nationals and immigrants residing in Qa$\operatorname{tar}$ [17]. For anti-SARS-CoV-2 antibodies, positive and negative anti-SARS-CoV-2 samples used in this study were randomly selected based on the results of our recent studies, where we used only male samples for studying SARS-CoV-2 seroprevalence among craft workers residing in Qatar [19]. 
Table 1. The outcome of SARS-CoV-2-lgG testing using the Mindray CL-900i assay on prepandemic sera that are both positive and negative for DENV-lgG

\begin{tabular}{lll}
\hline Mindray SARS-CoV-2-IgG & DENV antibody positive, $N(\%, 95 \% \mathrm{Cl})$ & DENV antibody negative, $N(\%, 95 \% \mathrm{Cl})$ \\
\hline Positive & $5(5.6,1.8-12.5)$ & $5(5.6,1.8-12.5)$ \\
Negative & $85(94.4,87.5-98.2)$ & $85(94.4,87.5-98.2)$ \\
\hline Total & 90 & 90 \\
\hline \multicolumn{1}{l}{$p$ value $=1.00}$. & \\
\hline
\end{tabular}

Detection of DENV-IgG by ELISA

The 182 postpandemic sera were tested for the presence of DENV-IgG using CE-certified commercial ELISA kits (NovaLisa $^{\circledR}$, dengue virus IgG; Ref. No. DENG0120, Germany). The microplate of this kit is coated with DENV type 2 antigens. The manufacturer reported diagnostic specificity and sensitivity of $98.0 \%$ (95\% CI: 89.35-99.95\%) and 100\% (95\% CI: 90.75-100.0\%), respectively. The detection was conducted per the manufacturer's instructions.

Detection of Anti-SARS-CoV-2-IgG by the CL-900i Automated Assay

The 180 prepandemic sera were tested for the presence of SARS-CoV-2-IgG using the CL-900i ${ }^{\circledR}$ SARS-CoV-2-IgG kit (Cat. No. SARS-CoV-2-IgG121, Mindray, China). The kit detects IgG antibodies to both the spike "S" and nucleocapsid " $N$ " proteins of SARS-CoV-2. The reported specificity and sensitivity of this kit were $95.3 \%$ (90.1-97.8) and 90.1\% (83.1-94.4), respectively [16].

\section{Statistical Analysis}

Serological results for SARS-CoV-2 and DENV were cross-tabulated. $\chi^{2}$ tests were conducted to compare the cross-reactivity between SARS-CoV-2 and DENV for both the CL-900i ${ }^{\circledR}$ SARSCoV-2-IgG kit and the NovaLisa DENV-IgG ELISA assay. The level of significance was indicated at $5 \%$.

\section{Results}

\section{CL-900i ${ }^{\circledR}$ Chemiluminescence Immunoassay}

Specificity/Cross-Reactivity with DENV-IgG

The 90 DENV-IgG-positive and 90 DENV-IgG-negative prepandemic sera samples were tested by the CL$900 \mathrm{i}^{\circledR}$ SARS-CoV-2-IgG kit (Table 1). The DENV-IgGpositive sera displayed 5 positives and 85 negatives for SARS-CoV-2-IgG. The DENV-IgG-negative sera also resulted in 5 positives and 85 negatives for SARS-CoV2-IgG. These results indicate no statistically significant difference in specificity (cross-reactivity) between the DENV-IgG-positive and DENV-IgG-negative sera ( $p$ value $=1.00)$.
Table 2. Outcome of DENV-IgG testing using the NovaLisa assay on postpandemic sera that are both positive and negative for SARSCoV-2-IgG

\begin{tabular}{|c|c|c|}
\hline $\begin{array}{l}\text { IgG DENV } \\
\text { ELISA }\end{array}$ & $\begin{array}{l}\text { SARS-CoV-2 antibody } \\
\text { positive, } N(\%, 95 \% \mathrm{Cl})\end{array}$ & $\begin{array}{l}\text { SARS-CoV-2 antibody } \\
\text { negative, } N(\%, 95 \% \mathrm{Cl})\end{array}$ \\
\hline Positive & $43(47.3,36.7-58.0)$ & $50(54.9,44.2-65.4)$ \\
\hline Negative & $47(51.6,40.9-62.3)$ & $40(44.0,33.6-54.8)$ \\
\hline Equivocal & $1(1.1,0.03-6.0)$ & $1(1.1,0.03-6.0)$ \\
\hline Total & 91 & 91 \\
\hline
\end{tabular}

DENV-IgG ELISA Cross-Reactivity with SARS-CoV$2-\operatorname{IgG}$

The 91 SARS-CoV-2-IgG-positive and 91 SARS-CoV2-IgG-negative postpandemic sera were tested by the NovaLisa DENV-IgG ELISA assay (Table 2). The SARSCoV-2-IgG-positive sera resulted in 43 positives, 47 negatives, and 1 equivocal for DENV-IgG. The SARS-CoV-2-IgG-negative sera resulted in 50 positives, 40 negatives, and 1 equivocal for DENV-IgG. These results indicate that there is no statistically significant difference in the proportion that is DENV-IgG-positive between the SARS-CoV-2-IgG-positive and SARS-CoV2 -IgG-negative sera ( $p$ value $=0.58)$.

\section{Discussion}

The assumption of cross-reaction between SARSCoV-2 and DENV was reported in several studies. However, most of these studies have built this assumption based on the antigenic cross-reactivity using the LFA and a few based on ELISA results detecting NS1 antigens [9-12]. Therefore, we investigated the possible serological cross-reaction between SARS-CoV-2 and DENV us- 
ing more advanced serological assays, which are the CL900i automated analyzer and the ELISA test.

A recent study utilized molecular docking and computational analysis to predict a significant surface interaction between DENV envelope (E) monoclonal antibodies and the SARS-CoV-2 receptor-binding domain of the S-protein [20]. Another study demonstrated that prior exposure to the dengue virus does not affect anti-N antibodies detection in COVID-19 patients using ELISA [21]. Taking into consideration the targeted antigen of both assays used in this study, the CL-900i targets both the $\mathrm{S}$ and $\mathrm{N}$ antigens of SARS-CoV-2, with $\mathrm{N}$ antigen as the main contributor for the kit according to the manufacturer's "personal communication." The NovaLisa DENV ELISA targets the whole virus antigens. Thus, this minimizes the possibility of cross-reactivity between the two viruses' antibodies when using CL-900i and DENV NovaLisa and could explain the similar results between the test groups and the control groups, with no evidence for a significant difference.

The DENV antibody positive and negative samples are prepandemic samples, hence eliminating the possibility of double infections. However, when tested by CL$900 \mathrm{i}$, there were five false positives in both groups. This cross-reactivity may be assay-specific; the specificity of CL-900i was reported to be $95.3 \%$ [16]. Hence, these DENV false-positive results in both the test and control groups may be due to different nonspecific cross-reactivity with other viruses, not necessarily reflecting SARSCoV-2 antibodies. On the other hand, the SARS-CoV-2 antibody positive and negative groups had 43 and 50 DENV false positives, respectively, and one equivocal each when tested using ELISA. This may be due to most patient serum used in this study are from the South Asian community, where it is hyperendemic to the DENV virus; thus, those samples might have prior exposure to DENV [22]. This is based on our previous observation of high seroprevalence of anti-dengue antibodies (74.8\%) in Qatar, especially among those from Asian Nationalities such as Philippines (95.8\%) and India (62.5\%) [17].

Our study results are in line with a preliminary study that investigated potential serological cross-reactivity between COVID-19 and dengue patients using ELISA and two LFA. Among the 32 positive SARS-CoV-2 sera tested by ELISA, no positive DENV IgG/IgM were observed, whereas among the 44 positive DENV sera, 1 false positive for SARS-CoV-2 resulted. This study concluded that there is a low risk of serological cross-reaction between the two viruses [13]. Furthermore, two studies have tested potential DENV cross-reactivity with SARS-CoV-2 using the automated analyzers, Abbott and Roche. Both Architect Abbott and Elecsys Roche anti-SARS-CoV-2 immunoassays used in these studies detect antibodies against the nucleocapsid $(\mathrm{N})$ antigen $[23,24]$. Out of 46 positive DENV samples tested by Abbott and 74 tests by Roche, the results showed zero cross-reactivity among the tested samples in both analyzers $[25,26]$. These latter studies, as well as our study, support the importance of using advanced diagnostic assays instead of rapid tests to avoid misdiagnosis, particularly in areas where DENV is endemic.

In conclusion, our results suggest that there is a low risk of serological cross-reactivity between anti-DENV2 total antibodies type 2 antigens (nonstructural protein, NS2) based on NovaLisa ELISA kit and anti-N and antiS SARS-CoV2 IgG detected by Mindray CL-900i. However, these results do not resolve all the concerns related to potential cross-reactivity between DENV and SARSCoV-2; thus, the results of this study should be interpreted with caution. However, it emphasizes the importance of using advanced serological analyzers to avoid false-positive results. This study has some limitations, CL- $900 i^{\circledR}$ detects IgG antibodies for both $\mathrm{S}$ and N proteins of SARS-CoV-2, and NovaLisa ELISA detects the positive IgG DENV virus antigen. Therefore, for future investigation, assays targeting different antigens and antibodies (IgG and IgM) for DENV and SARS-CoV-2 should be utilized. In addition, The DENV cohort sera used were confirmed by IgG serology testing only and not confirmed by PCR; thus, it would be more reliable to use samples that are DENV PCR confirmed positive to deduce a more decisive conclusion. Furthermore, the study was done on a limited number of samples from DENV hyperendemic regions; hence, it would be ideal for testing for DENV and SARS-CoV-2 cross-reactivity using patient samples that are not from DENV endemic regions.

\section{Acknowledgments}

The authors would like to thank Qatar National Library (QNL), a member of the Qatar Foundation, for sponsoring the publication fees of this article. The statements made herein are solely the responsibility of the authors. 


\section{Statement of Ethics}

This study protocol was reviewed and approved by Qatar University Institutional Review Board, approval numbers QU-IRB 1492-E/21 and QU-IRB 804-E/17. All participants have given their free and written informed consent before specimen collection and testing.

\section{Conflict of Interest Statement}

The authors declare no conflict of interest.

\section{Funding Sources}

L.J.A. acknowledges the support of the Biomedical Research Program and the Biostatistics, Epidemiology, and Biomathematics Research Core, both at Weill Cornell Medicine-Qatar. This work was made possible by Grant Nos. RRC-2-032 and UREP19-013-3001 from the Qatar National Research Fund (a member of Qatar Foundation). The statements made herein are solely the responsi- bility of the authors. In addition, G.K.N. would also like to acknowledge funds from Qatar University's internal Grant QUERGCMED-2020-2.

\section{Author Contributions}

Conceptualization: G.K.N. and H.M.Y. Methodology: F.M.S., F.H.A., D.W.A., and E.S.A. Formal analysis: F.M.S., F.H.A., E.S.A., L.J.A., and G.K.N. Validation: G.K.N. and F.M.S. Investigation: F.M.S., F.H.A., G.K.N., and L.J.A. Resources: G.K.N., D.W.A., and H.Q. Data curation: G.K.N. and F.M.S. Writing-original draft preparation: F.M.S., F.H.A., G.K.N., and L.J.A. Writing, review, and editing: G.K.N., H.M.Y., and L.J.A. Visualization: G.K.N. and F.M.S. Supervision: G.K.N. and F.M.S. Project administration: G.K.N. and D.W.A. Funding acquisition: G.K.N. and H.Q. All authors have read and agreed to the published version of the manuscript.

\section{Data Availability Statement}

Derived data supporting the findings of this study are available from the corresponding author upon request.

\section{References}

1 García-Montero C, Fraile-Martínez O, Bravo C, Torres-Carranza D, Sanchez-Trujillo L, Gómez-Lahoz AM, et al. An updated review of SARS-CoV-2 vaccines and the importance of effective vaccination programs in pandemic times. Vaccines. 2021;9(5):433.

2 Vojdani A, Vojdani E, Kharrazian D. Reaction of human monoclonal antibodies to SARS-CoV-2 proteins with tissue antigens: implications for autoimmune diseases. Front Immunol. 2020;11:617089.

3 Benvenga S, Guarneri F. Molecular mimicry and autoimmune thyroid disease. Rev Endocr Metab Disord. 2016;17(4):485-98.

4 Murugavelu P, Perween R, Shrivastava T, Singh V, Ahmad Parray H, Singh S, et al. Nonneutralizing SARS CoV-2 directed polyclonal antibodies demonstrate cross-reactivity with the HA glycans of influenza virus. Int Immunopharmacol. 2021;99:108020.

5 Lustig Y, Keler S, Kolodny R, Ben-Tal N, Atias-Varon D, Shlush E, et al. Potential antigenic cross-reactivity between SARS-CoV-2 and dengue viruses. Clin Infect Dis. $2021 \mathrm{Oct}$ 5;73(7):e2444.

6 Vicente CR, Herbinger KH, Fröschl G, Malta Romano C, de Souza Areias Cabidelle A, Cerutti Junior C. Serotype influences on dengue severity: a cross-sectional study on 485 confirmed dengue cases in Vitória, Brazil. BMC Infect Dis. 2016;16(1):1-7.

7 World Health Organization. Dengue and severe dengue; 2021. Available from: https:// www.who.int/news-room/fact-sheets/detail/ dengue-and-severe-dengue.
8 Nacher M, Douine M, Gaillet M, Flamand C, Rousset D, Rousseau C, et al. Simultaneous dengue and COVID-19 epidemics: difficult days ahead?. PLoS Negl Trop Dis. 2020;14(8): e0008426.

9 Harapan H, Ryan M, Yohan B, Abidin RS, Nainu F, Rakib A, et al. Covid-19 and dengue: double punches for dengue-endemic countries in Asia. Rev Med Virol. 2021;31(2): e2161.

10 Yan G, Lee CK, Lam LTM, Yan B, Chua YX, Lim AYN, et al. Covert COVID-19 and falsepositive dengue serology in Singapore. Lancet Infect Dis. 2020;20(5):536.

11 Nath H, Mallick A, Roy S, Sukla S, Basu K, De $A$, et al. Dengue antibodies can cross-react with SARS-CoV-2 and vice versa-antibody detection kits can give false-positive results for both viruses in regions where both COVID-19 and Dengue co-exist; 2020.

12 Oliveira BA, de Oliveira LC, de Oliveira FM, Pereira GM, de Souza RM, Manuli ER, et al. Evaluation of eleven immunochromatographic assays for SARS-CoV-2 detection: investigating dengue cross-reaction; 2020.

13 Spinicci M, Bartoloni A, Mantella A, Zammarchi L, Rossolini GM, Antonelli A. Low risk of serological cross-reactivity between dengue and COVID-19. Mem Inst Oswaldo Cruz. 2020;115:e200225.

14 Masyeni S, Santoso MS, Widyaningsih PD, Asmara DW, Nainu F, Harapan H, et al. Serological cross-reaction and coinfection of dengue and COVID-19 in Asia: experience from Indonesia. Int J Infect Dis. 2021;102:152-4.
15 García-Díaz G, Montalvo-Varela E, CanoPérez E, da Silva Francisco Junior R, Rodriguez-Morales A. Usefulness and limitations of implementing rapid tests for the diagnosis of COVID-19 in Latin America. Infez Med. 2020;28(4):642-4.

16 Younes S, Al-Jighefee H, Shurrab F, Al-Sadeq DW, Younes N, Dargham SR, et al. Diagnostic efficiency of three fully automated serology assays and their correlation with a novel surrogate virus neutralization test in symptomatic and asymptomatic SARS-COV-2 individuals. Microorganisms. 2021;9(2):245.

17 Humphrey JM, Al-Absi ES, Hamdan MM, Okasha SS, Al-Trmanini DM, El-Dous HG, et al. Dengue and chikungunya seroprevalence among Qatari nationals and immigrants residing in Qatar. PLoS One. 2019;14(1): e0211574.

18 Nasrallah GK, Dargham SR, Mohammed LI, Abu-Raddad LJ. Estimating seroprevalence of herpes simplex virus type 1 among different Middle East and North African male populations residing in Qatar. J Med Virol. 2018; 90(1):184-90.

19 Al-Thani MH, Farag E, Bertollini R, Al Romaihi HE, Abdeen S, Abdelkarim A, et al., editors. SARS-CoV-2 infection is at herd immunity in the majority segment of the population of Qatar. In: Open forum infectious diseases: Oxford University Press; 2021. 
20 Nath H, Mallick A, Roy S, Sukla S, Biswas S, Journal SB. Computational modelling supports that dengue virus envelope antibodies can bind to SARS-CoV-2 receptor binding sites: is pre-exposure to dengue virus protective against COVID-19 severity? Comput Struct Biotechnol J. 2021;19:459-66.

21 Lytton SD, Yeasmin M, Ghosh AK, Bulbul M, Hassan R, Molla M, et al. Detection of anti-nucleocapsid antibody in COVID-19 patients in Bangladesh is not correlated with previous dengue infection. Pathogens. 2021; 10(6):637.
22 Balmaseda A, Hammond SN, Tellez Y, Imhoff L, Rodriguez Y, Saborio SI, et al. High seroprevalence of antibodies against dengue virus in a prospective study of schoolchildren in Managua, Nicaragua. Trop Med Int Health. 2006;11(6):935-42.

23 Nasrallah GK, Dargham SR, Shurrab F, AlSadeq DW, Al-Jighefee $\mathrm{H}$, Chemaitelly $\mathrm{H}$, et al. Analytic comparison between three highthroughput commercial SARS-CoV-2 antibody assays reveals minor discrepancies in a high-incidence population. Sci Rep. 2021; 11(1):1-10.
24 Ismail A, Shurrab FM, Al-Jighefee HT, AlSadeq DW, Qotba H, Al-Shaar IA, et al. Can commercial automated immunoassays be utilized to predict neutralizing antibodies after SARS-CoV-2 infection? A comparative study between three different assays; 2021.

25 Lau C, Hoo S, Yew S, Ong S, Lum L, Heng P, et al. Evaluation of an electrochemiluminescent SARS-CoV-2 antibody assay. J Appl Lab Med. 2020;5(6):1313-23.

26 Lau CS, Oh HML, Hoo SP, Liang YL, Phua SK, Aw TC. Performance of an automated chemiluminescence SARS-CoV-2 IG-G assay. Clin Chim Acta. 2020;510:760-6. 